Internat. J. Math. \& Math. Sci.

Vol. 24, No. 6 (2000) 389-401

S0161171200002362

(c) Hindawi Publishing Corp.

\title{
DECOMPOSITION CONDITIONS FOR TWO-POINT BOUNDARY VALUE PROBLEMS
}

\author{
WENYING FENG
}

(Received 1 December 1998)

\begin{abstract}
We study the solvability of the equation $x^{\prime \prime}=f\left(t, x, x^{\prime}\right)$ subject to Dirichlet, Neumann, periodic, and antiperiodic boundary conditions. Under the assumption that $f$ can be suitably decomposed, we prove approximation solvability results for the above equation by applying the abstract continuation type theorem of Petryshyn on A-proper mappings.
\end{abstract}

Keywords and phrases. Boundary value problem, Fredholm operator, A-proper mapping, feebly $a$-solvable.

2000 Mathematics Subject Classification. Primary 47H09; Secondary 34B15.

1. Introduction. Let $f:[0,1] \times \mathbb{R}^{2} \rightarrow \mathbb{R}$ be a continuous function. The purpose of this paper is to establish some new existence results on the solvability of second order ODE's of the form

$$
x^{\prime \prime}=f\left(t, x, x^{\prime}\right)
$$

subject to one of the following boundary conditions:

$$
\begin{gathered}
x(0)=x(1)=0, \\
x^{\prime}(0)=x^{\prime}(1)=0, \\
x(0)=x(1), \quad x^{\prime}(0)=x^{\prime}(1), \\
x(0)=-x(1), \quad x^{\prime}(0)=-x^{\prime}(1) .
\end{gathered}
$$

The solvability of (1.1) subject to the above Dirichlet, Neumann, periodic, and antiperiodic boundary conditions has been extensively studied by many authors (see $[1,2,3,5,6,7,9,10])$. In a recent paper [2], a decomposition condition for $f$ is imposed to ensure the solvability of (1.1) with the boundary condition (1.2). The theorems of [2] were proved by using the transversality theorem.

In this paper, under the assumption that $f$ can be suitably decomposed, we shall apply the abstract continuation type theorem of Petryshyn on A-proper mappings to prove approximation solvability results for (1.1) with the boundary conditions (1.2), (1.3), (1.4), and (1.5). Approximation solvability includes the classical Galerkin method. One of our theorems includes the result of [2]. When $f$ is independent of $x^{\prime \prime}$, our results generalize the results of $[9,10]$ and show that certain restrictions imposed in $[9,10]$ are not needed in this case. 
Some examples show that our theorems permit the treatment of equations to which the results of $[2,3,7]$ do not apply.

2. Preliminaries. We recall the definition of the $A$-proper mapping which was introduced by Petryshyn (see [8]).

DefinITION 2.1. Let $X, Y$ be Banach spaces. Suppose that $\left\{X_{n}\right\} \subset X$ and $\left\{Y_{n}\right\} \subset Y$ are sequences of finite dimensional oriented spaces and $Q_{n}: Y \rightarrow Y_{n}$ is a linear projection for each $n \in \mathbb{R}^{\mathbb{N}}$, then the scheme $\Gamma=\left\{X_{n}, Y_{n}, Q_{n}\right\}$ is said to be admissible for maps from $X$ to $Y$ provided that $\operatorname{dim} X_{n}=\operatorname{dim} Y_{n}$ for each $n$, $\operatorname{dist}\left(x, X_{n}\right) \equiv$ $\inf \left\{\|x-v\|_{X}: v \in X_{n}\right\} \rightarrow 0$ as $n \rightarrow \infty$ for each $x$ in $X$, and $Q_{n} y \rightarrow y$ for each $y$ in $Y$. For a given map $T: D \subset X \rightarrow Y$ the equation

$$
T x=y
$$

is said to be feebly approximation-solvable ( $a$-solvable) relative to $\Gamma$ if there exists $N_{y} \in \mathbb{R}^{\mathbb{N}}$ such that the finite dimensional equation

$$
T_{n}(x)=Q_{n} y, \quad\left(x \in D_{n} \equiv D \cap X_{n}, T_{n}=\left.Q_{n} T\right|_{D_{n}}\right),
$$

has a solution $x_{n} \in D_{n}$ for each $n \geq N_{y}$ such that $x_{n_{j}} \rightarrow x \in D$ in $X$ and $T x=y$.

DEFINITION 2.2. $T$ is said to be A-proper relative to $\Gamma$ if $T_{n}: D_{n} \subset X_{n} \rightarrow Y_{n}$ is continuous for each $n \in \mathbb{R}^{\mathbb{N}}$ and if $\left\{x_{n_{j}} \mid x_{n_{j}} \in D_{n_{j}}\right\}$ is any bounded sequence in $X$ such that $T_{n_{j}}\left(x_{n_{j}}\right) \rightarrow g$ for some $g$ in $Y$, then there is a subsequence $\left\{x_{n_{k}}\right\}$ of $\left\{x_{n_{j}}\right\}$ and $x \in D$ such that $x_{n_{k}} \rightarrow x$ in $X$ and $T x=g$.

For (2.1) to be $a$-solvable relative to a given $\Gamma$ the operator $T$ has essentially to be A-proper relative to $\Gamma$ (see [5]).

Let $L: X \rightarrow Y$ be a Fredholm operator of index zero. It was shown in [8] that if $Y$ has an admissible scheme then an admissible scheme $\Gamma_{L}$ (depending on $L$ ) can be constructed such that $L$ is $A$-proper relative to $\Gamma_{L}$. Suppose that $X=\operatorname{ker}(L) \oplus X_{1}, Y=Y_{0} \oplus \operatorname{im}(L)$, where $\operatorname{dim} \operatorname{ker}(L)=\operatorname{dim} Y_{0}$. Let $Q$ be a projection of $Y$ onto $Y_{0}$ and assume that there exists a continuous bilinear form $[\cdot, \cdot]$ on $Y \times X$ mapping $(y, x)$ into $[y, x]$ such that $y \in \operatorname{im}(L)$ if and only if $[y, x]=0$ for every $x \in \operatorname{ker}(L)$.

Our results will be proved by applying the following abstract continuation type theorem for $A$-proper mappings.

THEOREM 2.3 (see [6, 7]). Let $L$ be a Fredholm operator of index zero and $N: X \rightarrow Y$ be a continuous nonlinear map. Suppose there exists a bounded open set $G \in X$ with $0 \in G$ such that

(1) $L-\lambda N: \bar{G} \rightarrow Y$ is A-proper relative to $\Gamma$ for each $\lambda \in[0,1]$ with $N(\bar{G})$ bounded.

(2) $L x \neq \lambda N x-\lambda y$ for $x \in \partial G$ and $\lambda \in(0,1]$.

(3) $Q N x-Q y \neq 0$ for $x \in \partial G \cap \operatorname{ker}(L)$.

(4) Either $[Q N x-Q y, x] \geq 0$ or $[Q N x-Q y, x] \leq 0$ for $x \in \partial G \cap \operatorname{ker}(L)$. Then the equation

$$
L x-N x=y
$$


is feebly a-solvable relative to $\Gamma$ and in particular it has a solution $x \in G$. If $x$ is the unique solution in $G$, then (2.3) is strongly a-solvable.

3. Existence results. We use P1, P2, P3, and P4 to denote (1.1) subject to the boundary condition (1.2), (1.3), (1.4), and (1.5), respectively. Our first three theorems deal with the simple case (3.1).

THEOREM 3.1. Let $f:[0,1] \times \mathbb{R}^{2} \rightarrow \mathbb{R}$ be continuous. Consider the following boundary value problem:

$$
x^{\prime \prime}=f\left(t, x, x^{\prime}\right), \quad x(0)=x(1)=0 .
$$

Assume that $f$ has the decomposition

$$
f(t, x, p)=g(t, x, p)+h(t, x, p)
$$

such that

(1) $\int_{0}^{1} x g\left(t, x, x^{\prime}\right) d t \geq 0$ for all $x \in C^{2}[0,1]$ with $x(0)=x(1)=0$,

(2) $|h(t, x, p)| \leq a|x|+b|p|$,

where $a>0, b>0$ and $a+b \pi<\pi^{2}$. Then (3.1) is feebly a-solvable in $C^{2}[0,1]$.

Proof. Let $X=C_{0}^{2}=\left\{x \in C^{2}[0,1], x(0)=x(1)=0\right\}$ endowed with the norm $\|x\|=\max \left\{\|x\|_{\infty},\left\|x^{\prime}\right\|_{\infty},\left\|x^{\prime \prime}\right\|_{\infty}\right\}$, where $\|x\|_{\infty}=\max _{t \in[0,1]}|x(t)|$. Let $\|\cdot\|_{2}$ be the usual norm of $L^{2}(0,1)$ and let $L: X \rightarrow C[0,1]$ be the linear operator defined by

$$
L x=x^{\prime \prime}, \quad \text { for } x \in X \text {. }
$$

Define $N: C^{1}[0,1] \rightarrow C[0,1]$ to be the nonlinear mapping

$$
N x(t)=f\left(t, x(t), x^{\prime}(t)\right)
$$

Let $J: C_{0}^{2} \rightarrow C^{1}[0,1]$ denote the compact natural embedding. Since $N J$ is compact, $L-\lambda N J: C_{0}^{2} \rightarrow C^{1}[0,1]$ is A-proper for each $\lambda \in[0,1]$, [5]. Also, $L$ is invertible, so by Theorem 2.3, the $a$-solvability of (3.1) follows provided there exists an open bounded set $G \subset C_{0}^{2}$ such that

$$
L x-\lambda N J x \neq 0, \quad \text { for }(x, \lambda) \in\left(C_{0}^{2} \cap \partial G\right) \times(0,1] .
$$

This is equivalent to proving the following subset of $C_{0}^{2}$ is bounded:

$$
U=\left\{x \in C_{0}^{2}, L x-\lambda N J x=0, \lambda \in(0,1]\right\} .
$$

Let $x \in U$, then

$$
x^{\prime \prime}=\lambda\left(g\left(t, x, x^{\prime}\right)+h\left(t, x, x^{\prime}\right)\right) .
$$


Applying Wirtinger's inequality [4]: $\|x\|_{2} \leq(1 / \pi)\left\|x^{\prime}\right\|_{2}$, we obtain

$$
\begin{aligned}
\left\|x^{\prime}\right\|_{2}^{2} & =-\int_{0}^{1} x x^{\prime \prime} d t \\
& =-\lambda \int_{0}^{1} x g\left(t, x, x^{\prime}\right) d t-\lambda \int_{0}^{1} x h\left(t, x, x^{\prime}\right) d t \\
& \leq-\lambda \int_{0}^{1} x h\left(t, x, x^{\prime}\right) d t \\
& \leq a \int_{0}^{1}|x|^{2} d t+b\left(\int_{0}^{1}|x|^{2} d t\right)^{1 / 2}\left(\int_{0}^{1}\left|x^{\prime}\right|^{2} d t\right)^{1 / 2} \\
& \leq \frac{a+b \pi}{\pi^{2}}\left\|x^{\prime}\right\|_{2}^{2} .
\end{aligned}
$$

By our assumption, $a+b \pi<\pi^{2}$, so $x^{\prime}=0$. Since $x \in C_{0}^{2}, x(t)=0$. This completes the proof.

REMARK 3.2. In the case $g\left(t, x, x^{\prime}\right)=r(x) x^{\prime}$, where $r$ is continuous and $r(x) \in$ $C^{1}[0,1]$, condition (1) of Theorem 3.1 is always satisfied, since $\int_{0}^{1} x r(x) x^{\prime} d t=0$ for all $x \in C_{0}^{2}$.

We use the following condition (see [2]) and Condition 3.4 for a continuous function $g:[0,1] \times \mathbb{R}^{2} \rightarrow \mathbb{R}$.

Condition 3.3. $|g(t, x, p)| \leq A(t, x) \omega\left(p^{2}\right)$ for all $(t, x, p) \in[0,1] \times \mathbb{R}^{2}$, where $A(t, x)$ is bounded on each compact subset of $[0,1] \times \mathbb{R}, \omega \in C\left(\mathbb{R}^{\mathbb{N}},(0,+\infty)\right)$ is nondecreasing and satisfies

$$
\int_{0}^{+\infty} \frac{d s}{\omega(s)}=\infty
$$

Condition 3.4. $|g(t, x, p)| \leq \sum_{i=1}^{r} B_{i}(t, x) \omega_{i}(p)$ for all $(t, x, p) \in[0,1] \times \mathbb{R}^{2}$, where $B_{i}(t, x)$ is bounded on compact subsets of $[0,1] \times \mathbb{R}$ and $\omega_{i}(p)$ are functions such that

$$
\int_{0}^{1}\left|x^{\prime}(t)\right|^{2} d t \leq M \Longrightarrow \int_{0}^{1}\left|\omega_{i}\left(x^{\prime}(t)\right)\right| d t \leq M_{0}
$$

where $M, M_{0}$ are constants, $M_{0}$ may depend on $M$.

The following theorem is a generalization of Theorem 1 in [2].

THEOREM 3.5. Let $f$ have the decomposition

$$
f(t, x, p)=g(t, x, p)+h(t, x, p) .
$$

Assume that

(1) $\int_{0}^{1} x g\left(t, x, x^{\prime}\right) d t \geq 0$ for all $x \in C_{0}^{2}$;

(2) $|h(t, x, p)| \leq a|x|+b|p|+\sum_{i=1}^{n} c_{i}|x|^{\alpha_{i}}+\sum_{j=1}^{m} d_{j}|p|^{\beta_{j}}$, where $a \geq 0, b \geq 0$, $0 \leq \alpha_{i}, \beta_{j}<1$

(3) $g(t, x, p)$ satisfies Condition 3.3 or Condition 3.4 .

Then (3.1) is feebly a-solvable in $C^{2}[0,1]$ provided that $a+b \pi<\pi^{2}$. 
Proof. By the same argument as in the proof of Theorem 3.1, we only need to prove that the set

$$
U=\left\{x \in C_{0}^{2}, L x-\lambda N J x=0, \lambda \in(0,1)\right\}
$$

is bounded. As in the proof of Theorem 3.1, for $x \in U$,

$$
\begin{aligned}
\left\|x^{\prime}\right\|_{2}^{2} & \leq \int_{0}^{1}\left|x h\left(t, x, x^{\prime}\right)\right| d t \\
& \leq \int_{0}^{1}|x|\left(a|x|+b\left|x^{\prime}\right|+\sum_{i=1}^{n} c_{i}|x|^{\alpha_{i}}+\sum_{j=1}^{m} d_{j}\left|x^{\prime}\right|^{\beta_{j}}\right) d t \\
& \leq a\|x\|_{2}^{2}+b\|x\|_{2}\left\|x^{\prime}\right\|_{2}+\sum_{i=1}^{n} c_{i}\|x\|_{2}\left(\int_{0}^{1}|x|^{2 \alpha_{i}}\right)^{1 / 2}+\sum_{j=1}^{m} d_{j}\|x\|_{2}\left(\int_{0}^{1}\left|x^{\prime}\right|^{2 \beta_{j}}\right)^{1 / 2} \\
& \leq\left(\frac{a}{\pi^{2}}+\frac{b}{\pi}\right)\left\|x^{\prime}\right\|_{2}^{2}+\sum_{i=1}^{n} \frac{c_{i}}{\pi}\left\|x^{\prime}\right\|_{2}\|x\|_{2}^{\alpha_{i}}+\sum_{i=j}^{m} \frac{d_{j}}{\pi}\left\|x^{\prime}\right\|_{2}\left\|x^{\prime}\right\|_{2}^{\beta_{j}} \\
& \leq\left(\frac{a}{\pi^{2}}+\frac{b}{\pi}\right)\left\|x^{\prime}\right\|_{2}^{2}+\frac{1}{\pi^{2}} \sum_{i=1}^{n} c_{i}\left\|x^{\prime}\right\|_{2}^{1+\alpha_{i}}+\frac{1}{\pi} \sum_{j=1}^{m} d_{j}\left\|x^{\prime}\right\|_{2}^{1+\beta_{j}} .
\end{aligned}
$$

Suppose that $\left\|x^{\prime}\right\|_{2} \neq 0$, since otherwise $x=0$. By our assumption $(a+b \pi) / \pi^{2}<1$, we have

$$
\left(1-\frac{a+b \pi}{\pi^{2}}\right)\left\|x^{\prime}\right\|_{2} \leq \frac{1}{\pi^{2}} \sum_{i=1}^{n} c_{i}\left\|x^{\prime}\right\|_{2}^{\alpha_{i}}+\frac{1}{\pi} \sum_{j=1}^{m} d_{j}\left\|x^{\prime}\right\|_{2}^{\beta_{j}} .
$$

If $\left\|x^{\prime}\right\|_{2} \rightarrow \infty$, we will have a contradiction since $0 \leq \alpha_{i}, \beta_{i}<1$. So there exists a constant $M>0$ such that $\left\|x^{\prime}\right\|_{2} \leq M$. This implies

$$
\|x\|_{\infty} \leq \int_{0}^{1}\left|x^{\prime}\right| d t \leq\left\|x^{\prime}\right\|_{2} \leq M .
$$

Suppose that $g$ satisfies Condition 3.3, then

$$
\left|x^{\prime \prime}\right| \leq A_{1} \omega\left(x^{\prime 2}\right)+C+b\left|x^{\prime}\right|+\sum_{j=1}^{m} d_{j}\left|x^{\prime}\right|_{j}^{\beta},
$$

where $A_{1}, C$ are positive constants. Since

$$
\left|x^{\prime}\right|^{\beta_{j}} \leq \frac{1}{2}\left(1+\left|x^{\prime}\right|^{2 \beta_{j}}\right) \leq 1+\left|x^{\prime}\right|^{2}
$$

we have

$$
\left|x^{\prime \prime}\right| \leq A_{1} \omega\left(x^{\prime 2}\right)+C+d\left(1+\left|x^{\prime}\right|^{2}\right) \leq A\left(\omega\left(x^{\prime 2}\right)+2+\left|x^{\prime}\right|^{2}\right),
$$

where $A=\max \left\{A_{1}, C, d\right\}$. As in the proof of Theorem 1 in [2], equation (3.18) implies that $\left|x^{\prime}\right|$ is bounded (for completeness, we give the proof here). Each $t \in[0,1]$ for which $x^{\prime}(t) \neq 0$ belongs to some interval $\left[s_{1}, s_{2}\right] \subset[0,1]$ with $x^{\prime}(t) \neq 0$ on $\left(s_{1}, s_{2}\right)$ 
and $x^{\prime}\left(s_{1}\right)=0$ or $x^{\prime}\left(s_{2}\right)=0$. Suppose that $x^{\prime}\left(s_{1}\right)=0$ and $x^{\prime}(t)>0$ on $\left(s_{1}, s_{2}\right)$. Define $z(t)=x^{\prime}(t), t \in\left[s_{1}, s_{2}\right]$. Then (3.18) implies that

$$
\frac{2 z(t) z^{\prime}(t)}{\omega\left(z^{2}(t)\right)+z^{2}(t)+2} \leq 2 A x^{\prime}(t), \quad t \in\left[s_{1}, s_{2}\right] .
$$

By integrating this inequality, we obtain

$$
\int_{0}^{z^{2}(t)} \frac{d s}{\omega(s)+s+2} \leq 4 A M, \quad t \in\left(s_{1}, s_{2}\right) .
$$

The assumption $\omega \in C\left(\mathbb{R}^{\mathbb{N}},(0,+\infty)\right)$ is nondecreasing and satisfies

$$
\int_{0}^{+\infty} \frac{d s}{\omega(s)}=\infty
$$

implies that (see [1]),

$$
\int_{0}^{\infty} \frac{d s}{\omega(s)+s+2}=\infty
$$

This ensures that there exists a constant $M_{1}>0$ such that $\left|x^{\prime}(t)\right| \leq M_{1}, t \in\left[s_{1}, s_{2}\right]$. Considering all the possible cases, we obtain that there exists a constant $M_{1}$ such that $\left\|x^{\prime}\right\|_{\infty} \leq M_{1}$. Let

$$
M_{2}=\sup _{t \in[0,1],|x| \leq M,|p| \leq M_{1}}|f(t, x, p)|,
$$

then $\|x\| \leq \max \left\{M, M_{1} M_{2}\right\}$. Hence, $U$ is bounded.

If $g$ satisfies Condition 3.4, then there exists $A_{2}>0$ such that

$$
\left|x^{\prime \prime}\right| \leq A_{2}\left(\sum_{i=1}^{r}\left|\omega_{i}\left(x^{\prime}\right)\right|+\left|x^{\prime}\right|^{2}+1\right) \text {. }
$$

Hence

$$
\int_{0}^{1}\left|x^{\prime \prime}\right| d t \leq A_{2}\left(\sum_{i=1}^{r} \int_{0}^{1}\left|\omega\left(x^{\prime}\right)\right| d t+\int\left|x^{\prime}\right|^{2} d t+1\right) \leq A_{2}\left(r M_{0}+M+1\right)=M_{3} .
$$

Suppose that $\xi \in[0,1]$ is such that $x^{\prime}(\xi)=0$. Then $x^{\prime}(t)=\int_{\xi}^{t} x^{\prime \prime}(s) d s$, and hence

$$
\left\|x^{\prime}\right\|_{\infty} \leq\left\|x^{\prime \prime}\right\|_{1} \leq M_{3} .
$$

This follows that $U$ is bounded.

REMARK 3.6. Theorem 1 in [2] is the special case of Theorem 3.5 when $a=0, b=0$, and $n=m=1$.

EXAMPLE 3.7. Consider the following boundary value problem:

$$
x^{\prime \prime}=x^{2 n+1} x^{\prime 2}+x^{\prime}-(x)^{1 / 3}, \quad x(0)=x(1)=0,
$$


where $n$ is a natural number. Let

$$
g(t, x, p)=x^{2 n+1} p^{2}, \quad h(t, x, p)=p-x^{1 / 3} .
$$

Then by Theorem 3.5 , this boundary value problem is feebly $a$-solvable in $C^{2}[0,1]$ and in particular it has a solution in $C^{2}[0,1]$.

Obviously, Theorem 1 in [2] cannot be applied to it. Also, we cannot find constants $M>0$ and $a, b \in \mathbb{R}$ such that

$$
x \geq M \Longrightarrow f(t, x, 0)>a \text { while } x \leq-M \Longrightarrow f(t, x, 0)<b
$$

since $f(t, x, 0) \rightarrow-\infty$ as $x \rightarrow \infty$ and $f(t, x, 0) \rightarrow \infty$ as $x \rightarrow-\infty$. Hence, Theorem 4.1 in [3] and Theorem 2.1 in [7] cannot be applied.

THEOREM 3.8. Let $f, g, h$ be as in Theorem 3.5 and instead of conditions (1) and (3), $g$ satisfies the following condition:

$$
p g(t, x, p) \leq 0, \quad \text { for }(t, x, p) \in[0,1] \times \mathbb{R}^{2} .
$$

Then (3.1) is feebly a-solvable in $C^{2}[0,1]$ provided that $a+b<1 / 2$.

Proof. Again we will prove that $U$ is bounded. Let $x \in U$, there exists $\xi \in(0,1)$ such that $x^{\prime}(\xi)=0$. Hence

$$
\begin{aligned}
\frac{1}{2}\left(x^{\prime}(t)\right)^{2} & =\int_{\xi}^{t} x^{\prime} x^{\prime \prime} d s \leq \lambda \int_{\xi}^{t} x^{\prime} h\left(s, x, x^{\prime}\right) d s \leq \int_{0}^{1}\left|x^{\prime}\right|\left|h\left(t, x, x^{\prime}\right)\right| d t \\
& \leq\left\|x^{\prime}\right\|_{\infty}\left(a\|x\|_{\infty}+b\left\|x^{\prime}\right\|_{\infty}+\sum_{i=1}^{n} c_{i}\|x\|_{\infty}^{\alpha_{i}}+\sum_{j=1}^{n} d_{j}\left\|x^{\prime}\right\|_{\infty}^{\beta_{j}}\right) .
\end{aligned}
$$

Suppose that $\left\|x^{\prime}\right\|_{\infty} \neq 0$, otherwise $x=0$. Since $a+b<1 / 2$ and

$$
\|x\|_{\infty} \leq\left\|x^{\prime}\right\|_{1} \leq\left\|x^{\prime}\right\|_{\infty}
$$

we obtain

$$
\left(\frac{1}{2}-a-b\right)\left\|x^{\prime}\right\|_{\infty} \leq \sum_{i=1}^{n} c_{i}\|x\|_{\infty}^{\alpha_{i}}+\sum_{j=1}^{m} d_{j}\left\|x^{\prime}\right\|_{\infty}^{\beta_{j}} .
$$

This implies that there exists $M>0$ such that $\left\|x^{\prime}\right\|_{\infty} \leq M$. By (3.32), $\|x\|_{\infty} \leq M$. Let

$$
M^{\prime}=\sup _{t \in[0,1],|x| \leq M,|p| \leq M}|f(t, x, p)|,
$$

then $\|x\| \leq \max \left\{M, M^{\prime}\right\}$. Thus $U$ is bounded.

Now, we consider P2, P3, and P4. These are resonance cases, since the linear part is noninvertible. In the following, let

$$
\begin{aligned}
& X_{i}=\left\{x \in C^{2}[0,1]: x \text { satisfies the boundary condition }(1 . i), i=2,3, \text { or } 4\right\}, \\
& U_{i}=\left\{x \in X_{i}: x^{\prime \prime}=\lambda f\left(t, x, x^{\prime}\right), \lambda \in(0,1]\right\},
\end{aligned}
$$

thus (1.1) subject to the boundary conditions (1.3), (1.4), and (1.5), respectively. 
THEOREM 3.9. Let $f:[0,1] \times \mathbb{R} \rightarrow \mathbb{R}$ be continuous. Assume that

$$
f(t, x, p)=g(t, x, p)+h(t, x, p),
$$

and $f, g$, and $h$ satisfy the following conditions:

(1) there exists a constant $M_{0}>0$ such that $x f(t, x, 0)>0$ for $|x|>M_{0}$;

(2) (a) $g$ satisfies Condition 3.3 or

(b) $g$ satisfies Condition 3.4 and $\int_{0}^{1} x g\left(t, x, x^{\prime}\right) d t \geq 0$ for all $x \in X_{i}$;

(3) $|h(t, x, p)| \leq C(t, x)+D(t, x)|p|^{2}+\sum_{j=1}^{n} d_{j}(t, x)|p|^{\beta_{j}}$, where $C(t, x), D(t, x)$, and $d_{j}(t, x)$ are bounded on compact subsets of $[0,1] \times \mathbb{R}$ and $0 \leq \beta_{j}<2$.

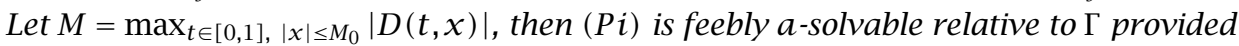
that $M_{0} M<1$.

Proof. Let $L: X_{i} \rightarrow C[0,1]$ be the linear operator defined by $L x=x^{\prime \prime}$. Then it is easily seen that $L$ is a Fredholm operator of index zero and $\operatorname{ker}(L)=\mathbb{R}$. Let $N x=$ $f\left(t, x, x^{\prime}\right)$ be the nonlinear map from $C^{1}[0,1]$ to $C[0,1]$ and $J_{i}: X_{i} \rightarrow C^{1}[0,1]$ be the compact continuous embedding. Then $L-\lambda N J_{i}$ is $A$-proper for each $\lambda \in[0,1]$. Moreover, let $Q y=\int_{0}^{1} y d t$ be the projection and

$$
[y, x]=\int_{0}^{1} y(t) x(t) d t
$$

be the bilinear form on $C[0,1] \times X_{i}$. For any $x \equiv c \in \operatorname{ker}(L)$, if $c>M_{0}$, then by assumption (1), $f(t, c, 0)>0$ and if $c<-M_{0}$, then $f(t, c, 0)<0$. Hence, $\|x\|=|c|>M_{0}$ implies $Q N J_{i} c \neq 0$. Assumption (1) also ensures that $\left[Q N J_{i} c, c\right] \geq 0$ for any $c \in \operatorname{ker}(L)$ with $|c|>M_{0}$. So, by Theorem 2.3 , to prove $(P i)$ is feebly $a$-solvable, we only need to prove $U_{i}$ is bounded.

Suppose that $x \in U_{i}$, Lemma 2.2 in [7] implies that $\|x\|_{\infty} \leq M_{0}$. Suppose $g$ satisfies 2(a), then by assumption (3), we obtain

$$
\begin{aligned}
\left|x^{\prime \prime}(t)\right| & \leq A(t, x) \omega\left(\left(x^{\prime}(t)\right)^{2}\right)+C(t, x)+D(t, x)\left|x^{\prime}(t)\right|^{2}+\sum_{j=1}^{n} d_{j}(t, x)\left|x^{\prime}(t)\right|_{\beta_{j}} \\
& \leq A_{1} \omega\left(\left(x^{\prime}(t)\right)^{2}\right)+C_{1}+M\left|x^{\prime}(t)\right|^{2}+\sum_{j=1}^{n} d_{j 1}\left(\left|x^{\prime}(t)\right|_{2}+1\right) \\
& \leq A_{2}\left(\omega\left(x^{\prime}(t)\right)^{2}+2+\left|x^{\prime}(t)\right|^{2}\right),
\end{aligned}
$$

where $A_{1}=\max _{t \in[0,1],|x| \leq M_{0}}|A(t, x)|, C_{1}, d_{j 1}$ are defined similarly and $A_{2}$ is a constant. As above, there exists $M_{1}>0$, such that $\left\|x^{\prime}\right\|_{\infty} \leq M_{1}$. This implies that $U_{i}$ is bounded.

Suppose that $g$ satisfies 2(b), then

$$
\begin{aligned}
\left\|x^{\prime}\right\|_{2}^{2} & =-\int_{0}^{1} x x^{\prime \prime} d t=-\lambda \int_{0}^{1} x g\left(t, x, x^{\prime}\right) d t-\lambda \int_{0}^{1} x h\left(t, x, x^{\prime}\right) d t \\
& \leq \int|x|\left|h\left(t, x, x^{\prime}\right)\right| d t \leq M_{0} \int_{0}^{1}\left(|C(t, x)|+D(t, x)\left|x^{\prime}\right|^{2}+\sum_{j=1}^{n} d_{j}(t, x)\left|x^{\prime}\right|^{\beta_{j}}\right) d t \\
& \leq M_{0} C^{\prime}+M_{0} M \int_{0}^{1}\left|x^{\prime}\right|^{2} d t+\sum_{j=1}^{n} d_{j}{ }^{\prime} \int_{0}^{1}\left|x^{\prime}\right|^{\beta_{j}} d t .
\end{aligned}
$$


Since $M_{0} M<1$, and by Holder's inequality,

$$
\int_{0}^{1}\left|x^{\prime}\right|^{\beta_{j}} d t \leq\left(\int_{0}^{1}\left|x^{\prime}\right|^{2} d t\right)^{\beta_{j} / 2}=\left\|x^{\prime}\right\|_{2}^{\beta_{j}}
$$

so

$$
\left(1-M_{0} M\right)\left\|x^{\prime}\right\|_{2}^{2} \leq M_{0} C^{\prime}+\sum_{j=1}^{n} d_{j}^{\prime}\left\|x^{\prime}\right\|_{2}^{\beta_{j}}
$$

This implies that there exists $M_{2}>0$ such that $\left\|x^{\prime}\right\|_{2} \leq M_{2}$ for $0 \leq \beta_{j}<2$. Since $g$ satisfies Condition 3.4, we obtain

$$
\int_{0}^{1}\left|x^{\prime \prime}(t)\right| d t \leq A \int_{0}^{1}\left|\omega\left(x^{\prime}\right)\right| d t+C^{\prime}+M \int_{0}^{1}\left|x^{\prime}\right|^{2} d t+\sum_{j=1}^{n} d_{j}^{\prime} \int_{0}^{1}\left(\left|x^{\prime}(t)\right|^{2}+1\right) \leq M_{3} .
$$

$x \in X_{i}$ implies that there exists $\xi \in[0,1]$ such that $x^{\prime}(\xi)=0$, hence

$$
\left\|x^{\prime}\right\|_{\infty}=\left\|\int_{\xi}^{t} x^{\prime \prime}(s) d s\right\|_{\infty} \leq\left\|x^{\prime \prime}\right\|_{1} \leq M_{3} .
$$

Thus, we have proved that $U_{i}$ is bounded, which completes the proof.

REMARK 3.10. In assumption (3) of Theorem 3.9, since $|p|^{\beta} \leq 1+|p|^{2}$, the third term is included in the first two terms, but it is convenient to make this split since the bound on the $|p|^{2}$ term only is important.

REMARK 3.11. In [10], the authors obtained the results on the existence of a solution to the following boundary value problem:

$$
\left(p(t) x^{\prime}\right)^{\prime}+\bar{f}\left(t, x, x^{\prime}, x^{\prime \prime}\right)=y(t), \quad x^{\prime}(0)=x^{\prime}(T)=0,
$$

and in [9] they studied the boundary value problem,

$$
x^{\prime \prime}+g_{1}(x) x^{\prime}+\bar{f}\left(t, x, x^{\prime}, x^{\prime \prime}\right)=y(t), \quad x(0)=x(1), \quad x^{\prime}(0)=x^{\prime}(1) .
$$

In (3.44), $p \in C^{1}[0, T]$ and $p_{0}=\min \{p(t) \mid 0 \leq t \leq T\}>0$. When $\bar{f}$ is independent of $x^{\prime \prime}$, let

$$
\bar{h}\left(t, x, x^{\prime}\right)=\bar{f}\left(t, x, x^{\prime}\right)-y(t),
$$

equation (3.44) can be rewritten in the following form (let $T=1$ ):

$$
x^{\prime \prime}=-\frac{p^{\prime}(t)}{p(t)} x^{\prime}-\frac{\bar{h}\left(t, x, x^{\prime}\right)}{p(t)}, \quad x^{\prime}(0)=x^{\prime}(1)=0 .
$$

To apply Theorem 3.9 to the boundary value problem (3.47), let

$$
g(t, x, p)=-\frac{p^{\prime}(t)}{p(t)} p, \quad h(t, x, p)=-\frac{\bar{h}(t, x, p)}{p(t)} .
$$


Then $g$ satisfies Condition 3.3 with $\omega(p)=p^{1 / 2}$. Assume that $|\bar{f}(t, x, p)| \leq A+B|x|+$ $C|p|$, since the condition (H4(i)) or (H4(ii)) of [10] implies assumption (1) of Theorem 3.9 , we obtain boundary value problem (3.47) is feebly $a$-solvable provided (H4(i)) or (H4(ii)) of [10] holds. Thus when $f$ does not depend on $x^{\prime \prime}$, in Theorem 2.1 in [10], the conditions $B T^{2}+\pi T\left(C+p_{1}\right) \leq \pi^{2} p_{0}$ of (H1) and (H2), (H3) are not necessary.

Similarly, when $\bar{f}$ is independent of $x^{\prime \prime}$, equation (3.45) can be rewritten as

$$
x^{\prime \prime}=-g_{1}(x) x^{\prime}-\bar{h}\left(t, x, x^{\prime}\right), \quad x(0)=x(1), \quad x^{\prime}(0)=x^{\prime}(1) .
$$

Let

$$
g(t, x, p)=-g_{1}(x) p, \quad h(t, x, p)=-\bar{h}(t, x, p) .
$$

Then $g$ satisfies Condition 3.4 since $\int_{0}^{1} x g_{1}(x) x^{\prime} d t=0$ for any $x \in X_{3}$. Assume that $|\bar{f}(t, x, p)| \leq A+B|x|+C|p|$, then condition (H4) of [9] ensures assumption (1) of Theorem 3.9. Applying Theorem 3.9, we obtain that boundary value problem (3.49) is feebly $a$-solvable provided (H4) of [9] holds. Hence in this case, in Theorem 2.1 in [9], the conditions $B+\pi C<2 \pi^{2}$ of (H1) and (H2), (H3) are not needed.

THEOREM 3.12. Let $f(t, x, p)=g(t, x, p)+h(t, x, p)$. Assume that

(1) there exists $M_{0}>0$ such that $x f(t, x, 0)>0$ for $|x|>M_{0}$;

(2) $p g(t, x, p) \geq 0$ or $p g(t, x, p) \leq 0$ for $(t, x, p) \in[0,1] \times \mathbb{R}^{2}$;

(3) $|h(t, x, p)| \leq C(t, x)+D(t, x)\left|x^{\prime}\right|+\sum_{j=1}^{n} d_{j}(t, x)\left|x^{\prime}\right|^{\alpha_{j}}$, where $C(t, x), D(t, x)$, and $d_{j}(t, x)$ are bounded on compact subsets of $[0,1] \times \mathbb{R}$ and $0 \leq \alpha_{j}<1$.

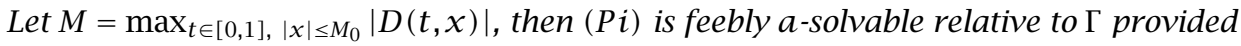
that $M<1 / 2$ if $p g(t, x, p) \leq 0$ and $M<1 / 4$ if $p g(t, x, p)>0$.

Proof. By the same argument with that in the proof of Theorem 3.9, we only need to prove $U_{i}$ is bounded. Let $x \in U_{i}$, then $\|x\|_{\infty} \leq M_{0}$ by Lemma 2.3 in [7]. Let $\xi \in[0,1]$ be such that $x^{\prime}(\xi)=0$, and assume that $p g(t, x, p)>0$ and $M<1 / 4$. Then

$$
\begin{aligned}
\frac{1}{2}\left(x^{\prime}(t)\right)^{2} & =\lambda \int_{\xi}^{t} x^{\prime} g\left(s, x, x^{\prime}\right) d s+\lambda \int_{\xi}^{t} x^{\prime} h\left(s, x, x^{\prime}\right) d s \\
& \leq \int_{0}^{1} x^{\prime} g\left(s, x, x^{\prime}\right) d s+\int_{0}^{1}\left|x^{\prime} h\left(s, x, x^{\prime}\right)\right| d s .
\end{aligned}
$$

Since $x \in X_{i}$, so

$$
\int_{0}^{1} x^{\prime} x^{\prime \prime} d t=\lambda \int_{0}^{1}\left(x^{\prime} g\left(t, x, x^{\prime}\right)+x^{\prime} h\left(t, x, x^{\prime}\right)\right) d t=0 .
$$

Hence,

$$
\frac{1}{2}\left(x^{\prime}(t)\right)^{2} \leq 2 \int_{0}^{1}\left|x^{\prime} h\left(s, x, x^{\prime}\right)\right| d t .
$$

Thus

$$
\begin{aligned}
\frac{1}{4}\left(x^{\prime}(t)\right)^{2} & \leq\left\|x^{\prime}\right\|_{\infty} \int_{0}^{1}\left(C(t, x)+D(t, x)\left|x^{\prime}\right|+\sum_{j=1}^{n} d_{j}(t, x)\left|x^{\prime}\right|^{\alpha_{j}}\right) d t \\
& \leq\left\|x^{\prime}\right\|_{\infty}\left(C^{\prime}+M\left\|x^{\prime}\right\|_{\infty}+\sum_{j=1}^{n} d_{j}^{\prime}\left\|x^{\prime}\right\|_{\infty}^{\alpha_{j}}\right) .
\end{aligned}
$$


Assume that $\left\|x^{\prime}\right\|_{\infty} \neq 0$, then

$$
\left(\frac{1}{4}-M\right)\left\|x^{\prime}\right\|_{\infty} \leq C^{\prime}+\sum_{j=1}^{n} d_{j}^{\prime}\left\|x^{\prime}\right\|_{\infty}^{\alpha_{j}} .
$$

Since $\alpha_{j}<1$, we obtain that there exists $M_{1}>0$ such that $\left\|x^{\prime}\right\|_{\infty} \leq M_{1}$. In the case $p g(t, x, p) \leq 0$ and $M<1 / 2$, instead of (3.51), we have

$$
\begin{aligned}
\frac{1}{2}\left(x^{\prime}(t)\right)^{2} & =\lambda \int_{\xi}^{t} x^{\prime} g\left(s, x, x^{\prime}\right) d s+\lambda \int_{\xi}^{t} x^{\prime} h\left(s, x, x^{\prime}\right) d s \\
& \leq \int_{0}^{1}\left|x^{\prime} h\left(s, x, x^{\prime}\right)\right| d s .
\end{aligned}
$$

So, by the same proof with above, there exists $M_{2}>0$ such that $\left\|x^{\prime}\right\|_{\infty} \leq M_{2}$. Thus in both cases, $U_{i}$ is bounded.

EXAMPLE 3.13. We study the following equation:

$$
x^{\prime \prime}= \pm x^{\prime 2 n+1}+Q(t, x)+\left|x^{\prime}\right|^{1 / 2}
$$

subject to the boundary conditions (1.3), (1.4), and (1.5), where $n$ is a natural number and $Q(t, x)$ is a continuous function. Assume that there exists $M_{0}>0, x Q(t, x)>0$ for $|x|>M_{0}$. By Theorem 3.12, the above boundary value problems is feebly $a$-solvable since $D(t, x)=0$. Since we cannot find $A(t, x)$ such that

$$
\left.\left| \pm p^{2 n+1}+Q(t, x)+\right| p\right|^{1 / 2} \mid \leq A(t, x) p^{2}+C(t, x),
$$

Theorem 2.1 in [7] and Theorem 4.1 in [3] cannot be used.

In our last theorem, we impose a condition which is similar to the condition (H3) of [10].

THEOREM 3.14. Let $f(t, x, p)=g(t, x, p)+h(t, x, p)$. Assume that

(1) there exists $M_{1}>0$ such that either $c f(t, c, 0) \geq 0$ for all $|c| \geq M_{1}$ or $c f(t, c, 0) \leq$ 0 for all $|c| \geq M_{1}$;

(2) there exists $M_{2}>0$ such that $\int_{0}^{1} f\left(t, x, x^{\prime}\right) d t \neq 0$ for $x \in X_{i}$ with $|x(t)|>M_{2}$ for $t \in[0,1]$

(3) $p g(t, x, p) \geq 0$ or $p g(t, x, p) \leq 0$ for $(t, x, p) \in[0,1] \times \mathbb{R}^{2}$;

(4) $|h(t, x, p)| \leq a|x|+b|p|+c|x|^{\alpha}+d|p|^{\beta}+e$, where $0 \leq \alpha, \beta<1$, and $a, b, c, d$,e are constants.

Then $(P i)$ is feebly a-solvable relative to $\Gamma$ provided that $a+b<1 / 2$ if $p g(t, x, p) \leq 0$ and $a+b<1 / 4$ if $p g(t, x, p)>0$.

Proof. Let $L, N, J_{i}, Q$ and the bilinear form $[y, x]$ be as in the proof of Theorem 3.9. For $c \in \operatorname{ker}(L)$, by assumption (2), $Q N c \neq 0$ if $|c| \geq M_{2}$. Moreover, according to assumption (1), [QNc,c] $\geq 0$ for all $|c| \geq M_{1}$ or $[Q N c, c] \leq 0$ for all $|c| \geq M_{1}$. Hence, by Theorem 2.3, $(P i)$ is feebly $a$-solvable if $U_{i}$ is bounded.

Let $x \in U_{i}$ and $\xi \in[0,1]$ be such that $x^{\prime}(\xi)=0$. By assumptions (3) and (4), using the same calculation with that in (3.51) and (3.56), we obtain that if $p g(t, x, p)>0$, 
then

$$
\frac{1}{4}\left\|x^{\prime}\right\|_{\infty}^{2} \leq\left\|x^{\prime}\right\|_{\infty}\left(a\|x\|_{\infty}+b\left\|x^{\prime}\right\|_{\infty}+c\|x\|_{\infty}^{\alpha}+d\left\|x^{\prime}\right\|_{\infty}^{\beta}+e\right)
$$

and if $p g(t, x, p) \leq 0$,

$$
\frac{1}{2}\left\|x^{\prime}\right\|_{\infty}^{2} \leq\left\|x^{\prime}\right\|_{\infty}\left(a\|x\|_{\infty}+b\left\|x^{\prime}\right\|_{\infty}+c\|x\|_{\infty}^{\alpha}+d\left\|x^{\prime}\right\|_{\infty}^{\beta}+e\right) .
$$

Assume that $\left\|x^{\prime}\right\|_{\infty} \neq 0$. Since $x \in X_{i}, N x \in \operatorname{im}(L)$, so $Q N x=0$. Assumption (2) ensures that there exists $\zeta \in[0,1]$ such that $|x(\zeta)| \leq M_{2}$. Writing $x(t)=\int_{\zeta}^{t} x^{\prime}(s) d s+$ $x(\zeta)$ gives

$$
\|x\|_{\infty} \leq\left\|x^{\prime}\right\|_{1}+M_{2} \leq\left\|x^{\prime}\right\|_{\infty}+M_{2}
$$

From the above discussion, in the case $p g(t, x, p)>0$, we obtain

$$
\left(\frac{1}{4}-a-b\right)\left\|x^{\prime}\right\|_{\infty} \leq M+c\left(\left\|x^{\prime}\right\|_{\infty}+M_{2}\right)^{\alpha}+d\left\|x^{\prime}\right\|_{\infty}^{\beta}+e .
$$

In the case $p g(t, x, p) \leq 0$, a similar inequality is obtained. These imply that there exists $M_{3}>0$ such that in both cases, $\left\|x^{\prime}\right\|_{\infty} \leq M_{3}$. By (3.61), $\|x\|_{\infty} \leq M_{3}$. Thus, we have proved that $U_{i}$ is bounded.

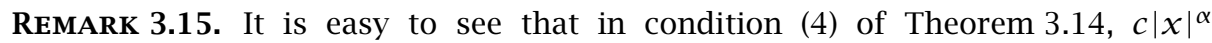
and $d|p|^{\beta}$ can, respectively, be replaced by $\sum_{i=1}^{n} c_{i}|x|^{\alpha_{i}}$ and $\sum_{j=1}^{m} d_{j}|p|^{\beta_{j}}$, where $0 \leq \alpha_{i}, \beta_{j} \leq 1$.

ACKNOwLedgement. I would like to express my thanks to Professor J. R. L. Webb for valuable discussions.

\section{REFERENCES}

[1] A. Constantin, Global existence of solutions for perturbed differential equations, Ann. Mat. Pura Appl. (4) 168 (1995), 237-299. MR 97a:34020. Zbl 847.34008.

[2] _ On a two-point boundary value problem, J. Math. Anal. Appl. 193 (1995), no. 1, 318-328. MR 96c:34032. Zbl 836.34021.

[3] A. Granas, R. B. Guenther, and J. W. Lee, On a theorem of S. Bernstein, Pacific J. Math. 74 (1978), no. 1, 67-82. MR 57\#10068. Zbl 377.34003.

[4] G. H. Hardy, J. E. Littlewood, and G. Polya, Inequalities, Cambridge Mathematical Library, Cambridge University Press, Cambridge, New York, 1988. MR 89d:26016. Zbl 634.26008 .

[5] W. V. Petryshyn, On the approximation-solvability of nonlinear equations, Math. Ann. 177 (1968), 156-164. MR 37\#2048. Zbl 162.20301.

[6] __ Some further results on periodic solutions of certain higher order nonlinear differential equations, Nonlinear Anal. 8 (1984), no. 9, 1055-1069. MR 86c:34077. Zbl 575.34031.

[7] Solvability of various boundary value problems for the equation $x^{\prime \prime}=$ $f\left(t, x, x^{\prime}, x^{\prime \prime}\right)-y$, Pacific J. Math. 122 (1986), no. 1, 169-195. MR 87g:34022. Zbl 585.34020.

[8] , Generalized Topological Degree and Semilinear Equations, Cambridge Tracts in Mathematics, 117, Cambridge University Press, Cambridge, 1995. MR 96k:47103. Zbl 834.47053. 
[9] W. V. Petryshyn and Z. S. Yu, Periodic solutions of nonlinear second-order differential equations which are not solvable for the highest derivative, J. Math. Anal. Appl. 89 (1982), no. 2, 462-488. MR 84c:34060. Zbl 516.34019.

[10] __ Solvability of Neumann BV problems for nonlinear second-order ODEs which need not be solvable for the highest-order derivative, J. Math. Anal. Appl. 91 (1983), no. 1, 244-253. MR 84g:34030. Zbl 513.34020.

Wenying Feng: Computer Science Studies Program, Trent University, Peterborough, ONTARIO, CANADA K9J 7B8

E-mail address: wfeng@trentu.ca 


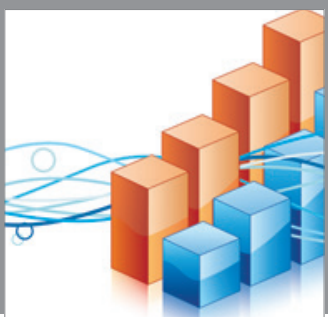

Advances in

Operations Research

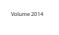

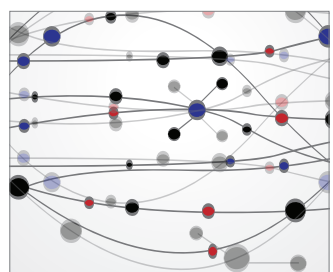

\section{The Scientific} World Journal
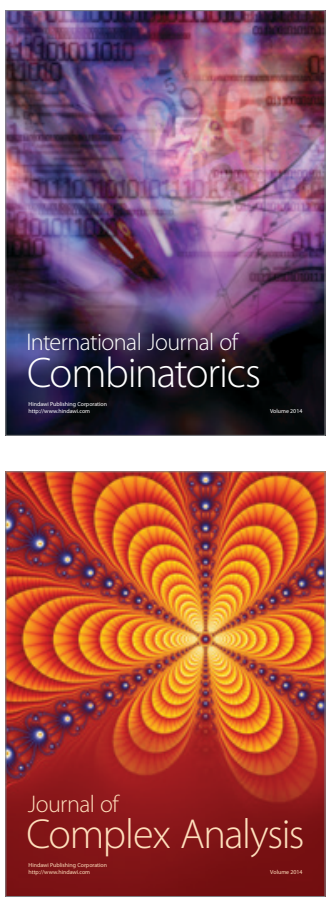

International Journal of

Mathematics and

Mathematical

Sciences
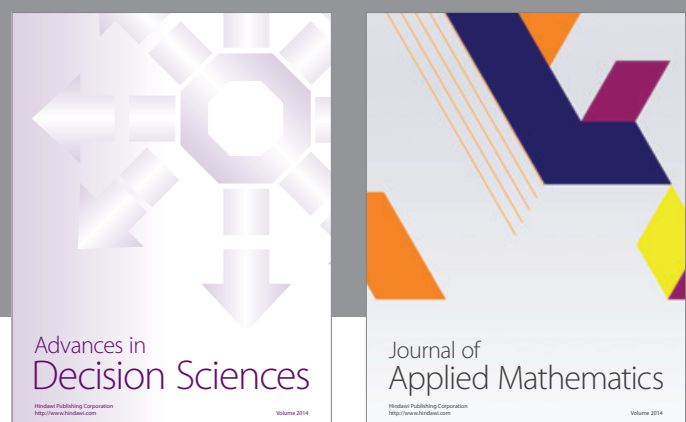

Journal of

Applied Mathematics
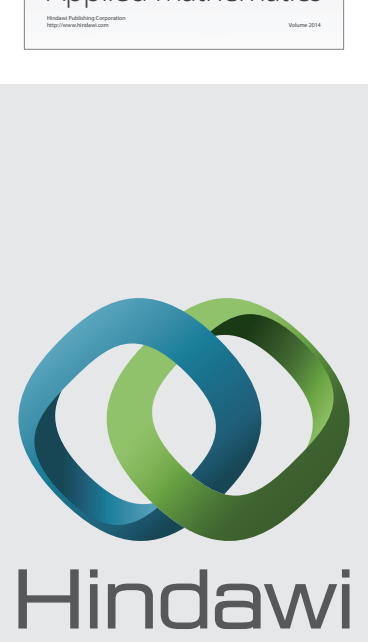

Submit your manuscripts at http://www.hindawi.com
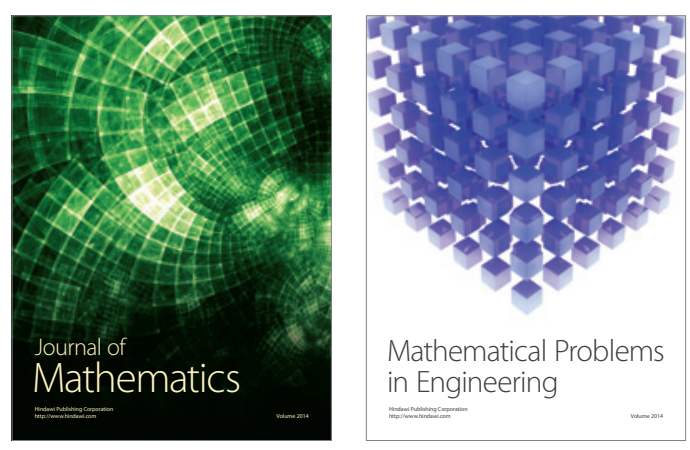

Mathematical Problems in Engineering
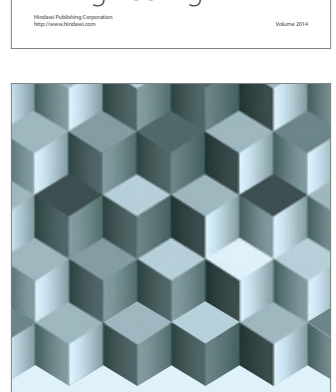

Journal of

Function Spaces
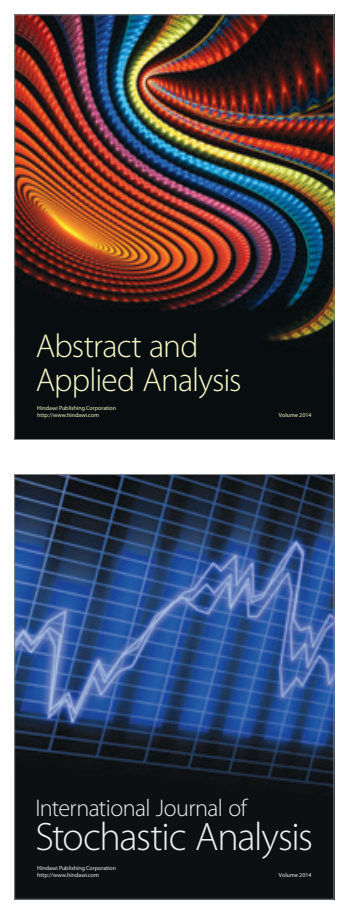

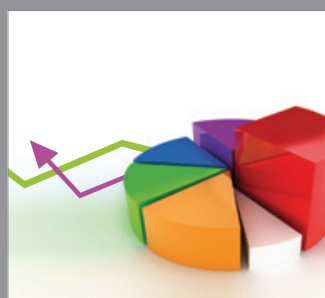

ournal of

Probability and Statistics

Promensencen
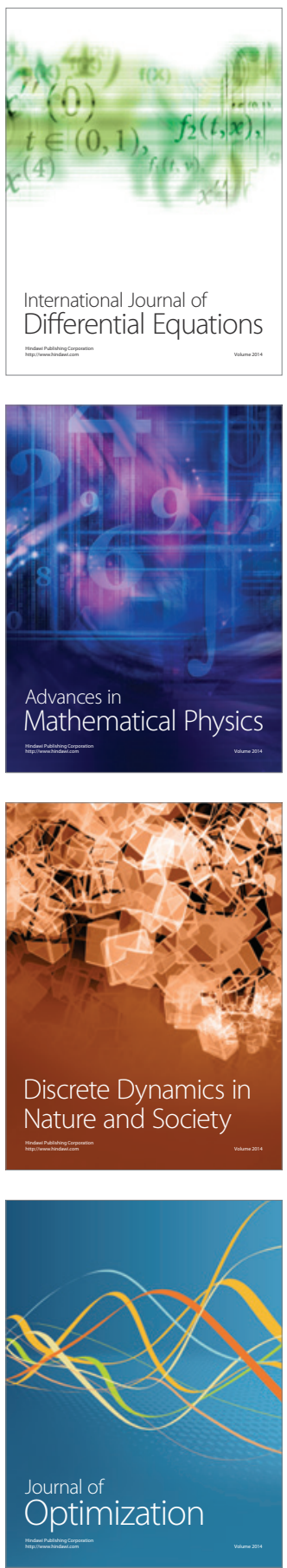